\title{
FRACTURAS METAFISARIAS DISTALES EXTRAARTICULARES DE TIBIA: PLACA PERCUTÁNEA VS. CLAVO ENDOMEDULAR
}

Wilson A. Restrepo MD*, Víctor A. Vargas MD*, Carlos Mario Olarte MD**, Juan Manuel Nossa MD***, Miguel Álvaro Triana MD****

\section{Resumen}

En el manejo de las fracturas diafisiarias distales de tibia aún existe controversia acerca de cuál técnica quirúrgica ofrece mayores beneficios. El objetivo de este trabajo es describir y comparar las utilizadas, clavo endomedular y placa percutánea, y los resultados en cuanto a consolidación y complicaciones, en pacientes tratados entre enero 2004 y mayo 2008 en el Hospital de San José y una institución asociada de tercer nivel de atención. Se describe una cohorte histórica de 55 pacientes con seguimiento a doce meses. El 76,4\% de la población fue de sexo masculino con promedio de edad de 36 años. Se observó mayor porcentaje de mala unión en los pacientes intervenidos con CE (10,7\% vs. 7,4\%, RR 1.45). La consolidación se presentó en el 92,9\% (26/28) de los pacientes manejados con CE contra un 88,9\% (24/27) de aquellos con PP. La pseudoartrosis se encontró en dos tratados con CE y en tres con PP (7,1\% vs. 11,1\%, RR 0.64). La falla del material de osteosíntesis no se observó con el uso de CE, mientras que dos con PP presentaron el evento ( $0 \%$ vs. 7,4\%). En cada grupo se vieron dos casos de infección (7,1\% vs. 7,4\%). Se requirió reintervención en $17,9 \%(5 / 28)$ con $\mathrm{CE}$ y en $25,9 \%(7 / 27)$ con PP. El manejo con CE aunque muestra un índice de mala unión mayor que con empleo de PP, evidenciaría un porcentaje de consolidación mayor y unos de complicaciones y reintervención menores. Se requieren estudios futuros multicéntricos para demostrar diferencias entre los dos tipos de tratamientos.

Palabras clave: fractura, tibia distal, clavo, placa, mala unión, pseudoartrosis, reintervención, infección, consolidación.

Abreviaturas: CE, clavo endomedular; PP, placa percutánea.

\section{TREATMENT OF DISTAL TIBIAL EXTRA-ARTICULAR METAPHYSIAL FRACTURES: PLATING VS. INTRAMEDULLARY NAILING}

\section{Abstract}

There is still controversy regarding which surgical technique is the most beneficial to manage distal tibial dia-physeal fractures. This paper describes and compares intramedullary nailing and percutaneous plating and results as to consolidation and complications in patients treated between January 2004 and May 2008 at the San José Hospital and at an associate tertiary level health care facility. A 55-patient historical cohort and 12-month follow-up was described.

The mean age was 36 years and $76.4 \%$ were male patients. A greater incidence of mal-union was observed in those who underwent an intramedullary nailing (10.7\% vs 7.4\%, RR 1.45$)$. Consolidation was achieved in $92.9 \%$ (26/28) of patients

Fecha recibido: febrero 18 de 2009 - Fecha aceptado: marzo 12 de 2009

* Residentes IV Ortopedia y Traumatología, Hospital de San José. Fundación Universitaria de Ciencias de la Salud, Bogotá D.C. Colombia.

** Instructor Asociado Clínica de Trauma, Hospital de San José, Fundación Universitaria de Ciencias de la Salud, Bogotá D.C. Colombia.

\footnotetext{
*** Instructor Asistente, Clínica Trauma y Cadera, Hospital de San José, Fundación Universitaria de Ciencias de la Salud, Bogotá D.C. Colombia.

**** Instructor Asociado, Ortopedia y Traumatología, Fundación Cardio Infantil de Bogotá D.C. Colombia.
} 
who received nailing compared to $88.9 \%(24 / 27)$ of those managed with plating. Pseudoarthrosis was present in 2 patients treated with a nail fixation and in 3 patients treated with a plate $(7.1 \%$ vs $11.1 \%$, RR 0.64$)$. Failure of the osteosynthesis material was not observed with the use of nailing while two patients with plating presented this type of event ( $0 \%$ vs $7.4 \%)$. Two cases of infection presented in each group ( $7.1 \%$ vs $7.4 \%)$. A second surgical intervention was necessary in $17.9 \%(5 / 28)$ of patients with nailing and in $25.9 \%(7 / 27)$ of those in the plating group.

Although management with intramedullary nailing shows a greater incidence of mal-union compared to plating, it would evidence a greater percentage of consolidation and less complications and need of a second procedure. Further multicentric trials are required in order to demonstrate the differences between these two treatment modalities.

Key words: fracture, distal tibia, nail, plate, mal-union, pseudoarthrosis, second procedure, infection, consolidation.

\section{Introducción}

El tratamiento de las fracturas diafisiarias distales de tibia es complejo y controversial. En la actualidad el de elección es el enclavamiento endomedular. ${ }^{1}$ Sin embargo, las fracturas del tercio distal de la tibia no tienen esta indicación especificada, pues presentan mayor riesgo de mala unión con tasas que van del $5 \%$ al $58 \% .^{2}$ La información en la literatura especializada no dilucida esto con claridad y los estudios en donde se compara el manejo de las fracturas de tibia distal, muestran un bajo nivel de evidencia. Zelle y colaboradores ${ }^{2}$ en una revisión sistemática de 1.125 fracturas de tibia distal no hallaron una diferencia significativa por la variedad en la metodología, presentando estudios observacionales con múltiples diferencias en los grupos de pacientes y en las indicaciones de cada tratamiento. Se registran fracturas abiertas en $28 \%$ con CE y un $4,9 \%$ en manejo con PP, sabiendo de antemano que esta condición es un factor determinante en el pronóstico de las fracturas diafisiarias de tibia. ${ }^{1,2}$

Im y colaboradores ${ }^{3}$ en un ensayo clínico aleatorio compararon el enclavamiento endomedular con reducción abierta y la fijación interna con placa utilizando técnica abierta, en el tratamiento de fracturas distales de tibia, concluyendo que es ventajoso el uso de CE cuando el trauma de tejidos blandos es severo y se puede tener mejor resultado con el uso de PP en lo referente al alineamiento postoperatorio. ${ }^{3}$ Cabe anotar que en este estudio se realizó la comparación utilizando técnica abierta con PP. Es importante aclarar que en la actualidad el procedimiento indicado para el manejo de fracturas diafisiarias distales de tibia con PP es la mínimamente invasiva (MIPPO) $)^{4,5}$ dada su ventaja desde el punto de vista de conservación biológica, requerida por la anatomía subcutánea de la tibia y por la especial distribución anatómica de su circulación, que se afecta en mayor forma con la técnica abierta de osteosíntesis. ${ }^{6,7}$

Por tanto, no existen hoy estudios comparativos de alto nivel de evidencia que describan la superioridad entre el CE o PP con técnica mínimamente invasiva. ${ }^{1,8}$ Revisando la estadística específica de las instituciones incluidas en el estudio en un lapso de tres años, se trataron 253 pacientes con fracturas diafisiarias de tibia. Es importante referir que dichas fracturas no estaban especificadas, pero se considera que podrían corresponder de 20 a $30 \%$ del total de las fracturas de tibia, lo que evidencia una incidencia real baja. La decisión en estas instituciones de usar clavo o placa se basa en la experiencia y preferencia del cirujano, considerando el estado de los tejidos blandos e inclinándose por el $\mathrm{CE}$ en pacientes con trauma de mayor energía y lesión severa de tejidos blandos, y utilizar la PP cuando hay trauma leve de tejidos blandos, y evidencia de fractura simple.

El objetivo del presente estudio es describir si existe diferencia entre los dos tipos de tratamiento quirúrgico en cuanto a consolidación, alineación, infección y reintervenciones en el seguimiento. Además, se busca describir factores como fractura ipsilateral de peroné, trauma de tejidos blandos y clasificación de la fractura.

\section{Métodos}

Se realizó un estudio tipo cohorte histórica, con seguimiento a tres, seis y doce meses a pacientes con fracturas diafisiarias distales extraarticulares de tibia, manejados mediante cirugía entre enero 2004 y mayo 2008 en el Hospital de San José y una institución asociada de tercer nivel de atención en Bogotá, que cumplieran con 
los siguientes criterios de inclusión: mayores de 18 años, que al ingreso presentaran fractura de tibia distal extraarticular, trazo de fractura por debajo de la unión del tercio medio con el tercio distal de la diáfisis y vértice distal del foco de fractura por encima de $4 \mathrm{~cm}$ de la superficie articular de la tibia distal y hasta $11 \mathrm{~cm}$ proximales, fracturas cerradas y abiertas hasta el grado III A de la clasificación de Gustilo y Anderson y pacientes a quienes como manejo primario se les realizara osteosíntesis con CE o PP. Se excluyeron los pacientes con manejo quirúrgico previo en otra institución, aquellos que requirieron manejo inicial con fijación externa antes del tratamiento y los que presentaron fracturas patológicas.

Los datos se obtuvieron de fuente secundaria acudiendo a registros de historia clínica y de fuente primaria en la evaluación de las radiografias prequirúrgicas y de los controles determinados. Para asegurar la calidad del dato, sólo los investigadores realizaron la recolección previo entrenamiento en la aplicación del instrumento. Los registros se obtuvieron a partir de la estadística de cada servicio, con diagnóstico de fracturas de tibia y manejadas con osteosíntesis. Se aplicaron filtros para identificar en cada historia clínica los candidatos para inclusión por el diagnóstico de fractura diafisiaria y manejo quirúrgico. Cumplieron los criterios 55 pacientes que ingresaron a la cohorte. Se evaluaron las radiografías preoperatorios y los hallazgos radiológicos al primero, tercero, sexto y duodécimo meses, que fueron interpretados por dos observadores independientes. En caso de discrepancia se realizó consenso para la determinación definitiva.

Se analizaron variables de edad y sexo, mecanismo causal de la fractura, trazo de fractura determinado en las radiografías prequirúrgicas y estadificado por la clasificación AO, el estado de los tejidos blandos según la clasificación de Gustilo y Anderson para las fracturas abiertas y la clasificación de Tsherne para las cerradas, existencia de lesiones asociadas, presencia de fractura de peroné, su nivel y la realización o no de osteosíntesis, las características básicas de los implantes utilizados y el tiempo en minutos del procedimiento en el registro anestésico.

Se evaluaron las siguientes variables de desenlace: mala unión al observar una angulación mayor de $5^{\circ}$ en cual- quiera de los planos del eje anatómico evidenciada en cualquiera de las valoraciones radiológicas, retardo de consolidación en ausencia de signos radiológicos al tercer mes y pseudoartrosis en ausencia de signos radiológicos de unión a los seis meses postoperatorios. La variable consolidación se evaluó por la presencia de callo óseo visible en el foco de fractura, con más de tres corticales continuas, independiente de si presentó retardo de consolidación o pseudoartrosis, y si requirió procedimientos adicionales. La presencia de falla en el material de osteosíntesis se determinó por alteraciones mecánicas del implante registradas en la historia clínica. La infección fue definida como la presencia de cultivos positivos de la herida quirúrgica y el requerimiento de procedimientos adicionales.

Se realizó una técnica estándar para enclavamiento endomedular con abordaje parapatelar medial o transpatelar a nivel de rodilla, utilización de pinzas para mantener la reducción o tornillos polares si no se obtuviese dicha reducción, rimado endomedular y bloqueos proximales guiados, $\mathrm{y}$ bloqueos distales a mano alzada.

Para la técnica de PP se realizó abordaje distal mínimamente invasivo, placa deslizada subcutánea y tornillos percutáneos proximales. La fijación del peroné se realizó con placa tercio de caña en los casos en que se consideró inestabilidad de la sindesmosis. Se utilizó material de osteosíntesis de Synthes (Synmed Quirúrgica S.A Synthes Colombia S.A). En ambos grupos se efectuó inmovilización con férula posterior de yeso por una semana, retiro de puntos, inicio de fisioterapia y apoyo parcial progresivo, considerando la progresión de la consolidación. Los pacientes fueron evaluados en la primera y cuarta semanas y tercero, sexto y duodécimo meses, con controles radiográficos en vistas AP y lateral secuenciales.

Las variables de estudio se resumieron utilizando la media y la desviación estándar para los datos continuos o la mediana cuando lo requirieron, y los recuentos o porcentajes para los datos categóricos. La estadística descriptiva se empleó para determinar las características de la población de acuerdo con los datos demográficos y clínicos, y para informar la incidencia de mala unión, pseudoartrosis, consolidación y complicaciones. Se re- 
portan medidas de asociación (RR) para comparar desenlaces dicótomos entre expuestos y no expuestos (consolidación, pseudoartrosis, mala unión, ISO). Todos los datos obtenidos se registraron en una base de datos (Excel, Microsoft 2007) y sus análisis se realizaron con el programa STATA versión $10 \circledR$ El protocolo fue aprobado por los comités de ética e investigación de la Fundación Universitaria de Ciencias de la Salud.

\section{Resultados}

Se incluyeron en el estudio 55 pacientes de los cuales 42 eran de sexo masculino y 13 femenino. Con respecto al tipo de osteosíntesis, en 28 se utilizó CE y en 27 PP. La distribución según la clasificación de la fractura $(\mathrm{AO})$ y el tipo de fractura (Gustilo - Anderson y Tsherne), se observan en la Tabla 1.

\section{Tabla I. Características de la población de estudio}

\begin{tabular}{|c|c|c|c|c|}
\hline \multirow{2}{*}{$\begin{array}{l}\text { Variable } \\
\text { Edad (mediana, RIQ) }\end{array}$} & \multicolumn{2}{|c|}{$\begin{array}{c}\text { Clavo } \\
\text { endomedular } \\
(n=28)\end{array}$} & \multicolumn{2}{|c|}{$\begin{array}{l}\text { Placa } \\
\text { percutánea } \\
(n=27)\end{array}$} \\
\hline & 30 & $(25-44)$ & 42 & $(3 I-5 I)$ \\
\hline Sexo masculino $(n, \%)$ & 21 & $(75)$ & 21 & $(77,8)$ \\
\hline Sexo femenino $(n, \%)$ & 7 & $(25)$ & 6 & $(22,2)$ \\
\hline \multicolumn{5}{|l|}{ Clasificación $\mathrm{AO} \ddagger(\mathrm{n}, \%)$} \\
\hline $42 \mathrm{Al}$ & 4 & $(\mid 4,2)$ & 7 & $(25,9)$ \\
\hline $42 \mathrm{~A} 2$ & 8 & $(28,5)$ & 11 & $(40,7)$ \\
\hline $42 \mathrm{~A} 3$ & 3 & $(10,7)$ & 3 & $(I I, I)$ \\
\hline $42 \mathrm{BI}$ & 3 & $(10,7)$ & 2 & $(7,4)$ \\
\hline 42B2 & 7 & $(25)$ & 3 & $(I I, I)$ \\
\hline $43 \mathrm{~B} 3$ & 2 & $(7,1)$ & 1 & $(3,7)$ \\
\hline $43 \mathrm{Cl}$ & 1 & $(3,6)$ & 0 & $(0)$ \\
\hline \multicolumn{5}{|l|}{ Tipo de fractura Gustilo \& Anderson (n,\%) } \\
\hline Abierta GI & 5 & $(17,9)$ & 4 & $(\mid 4,8)$ \\
\hline Abierta GII & 6 & $(21,4)$ & 1 & $(3,7)$ \\
\hline Abierta G IIIA & 1 & $(3,6)$ & 1 & $(3,7)$ \\
\hline Cerrada Tsherne 0 & 0 & $(0)$ & 0 & $(0)$ \\
\hline Cerrada Tsherne I & $\mathrm{I}$ & $(3,6)$ & $\mathrm{I}$ & $(3,7)$ \\
\hline Cerrada Tsherne 2 & 14 & $(50)$ & 18 & $(66,7)$ \\
\hline Cerrada Tsherne 3 & 1 & $(3,6)$ & 2 & $(7,4)$ \\
\hline \multicolumn{5}{|l|}{ Mecanismo de trauma $(n, \%)$} \\
\hline Accidente automovilístico & 12 & $(42,9)$ & 5 & $(\mid 8,5)$ \\
\hline Caída de altura & 4 & $(14,3)$ & 7 & $(25,9)$ \\
\hline Trauma en actividad deportiva & 3 & $(10,7)$ & 13 & $(47, I)$ \\
\hline Otros & 9 & $(32, I)$ & 2 & $(7,4)$ \\
\hline Fractura asociada de peroné $(n, \%)$ & 17 & $(60,7)$ & 20 & (74) \\
\hline Osteosíntesis peroné $(\mathrm{n}, \%)$ & 5 & $(29,4)$ & 5 & $(23,8)$ \\
\hline Tiempo cirugía en minutos (mediana, RIQ) & 102 & $(87-122)$ & 120 & $(100-170)$ \\
\hline
\end{tabular}

$\ddagger$ AO: Clasificación AO Muller 
El 69\% de las fracturas presentaron lesiones asociadas y de éstas la más frecuente fue la fractura de peroné ipsilateral ( $\mathrm{n}=37), 26$ en el tercio medio y distal, de las cuales a diez (27\%) se les realizó manejo con osteosíntesis. En los tratados con CE $(n=28)$ se utilizó un diámetro promedio de $11 \mathrm{~mm} .{ }^{9}$ En el $44 \%$ se utilizó bloqueo dinámico y en el 56\% estático. Sólo dos requirieron tornillos polares para reducción definitiva. El $57,2 \%$ eran fracturas cerradas $(50 \%$ consideradas Tsherne II) y el restante $42,8 \%$ eran abiertas con predominio de grados I y II.

En $82,5 \%$ de los tratados con PP se utilizó placa LCP y en los demás la DCP o LC-DCP. El número de tornillos promedio utilizados fue de 6.6. E1 77,8\% eran fracturas cerradas y la mayor parte $(66,7 \%)$ estaban clasificadas como Tsherne II. El restante 22,2\% eran fracturas abiertas en mayor porcentaje grado I. Los resultados relacionados con las variables de desenlace, se presentan en la Tabla 2.

Se presentó mala unión en tres $(10,7 \%)$ de los intervenidos con CE, mientras que con PP se observó en dos
$(7,4 \%)$. Llama la atención que a los primeros que se manejaron con clavo y mostraron mala unión, no se les realizó osteosíntesis de peroné.

Se observó pseudoartrosis a los seis meses en dos (7,1\%) operados con CE y en $3(11,1 \%)$ con PP. Es importante mencionar que en este grupo las fracturas cerradas se consideraron Tsherne II ó III y estaban clasificados según la AO como fracturas tipo A o de baja complejidad. Ocurrió falla del material de osteosíntesis en dos operados con placa, en ambos casos de tipo DCP. Se presentó infección en cuatro casos, dos en cada tipo de intervención y todos menores de 50 años. La distribución fue igual para pacientes con fracturas abiertas y cerradas.

En cuanto a reintervención, cinco con $\mathrm{CE}(17,9 \%)$ requirieron procedimiento adicional y siete $(25,9 \%)$ de los manejados con PP. Un mayor porcentaje de pacientes en los cuales se utilizó CE y requirieron reintervención eran fracturas abiertas, mientras que con PP eran fracturas cerradas. De los cinco reintervenidos con $\mathrm{CE}$, a dos se les realizó lavado y desbridamiento por infección,

\begin{tabular}{|c|c|c|c|}
\hline Variable & $\begin{array}{c}\text { Clavo } \\
\text { endomedular }\end{array}$ & $\begin{array}{c}\text { Placa } \\
(n=28)\end{array}$ & $\begin{array}{c}R R^{*} \\
(n=27)\end{array}$ \\
\hline Mala unión (n,\%) & $3(10,7)$ & $2(7,4)$ & $\mathrm{I}, 45$ \\
\hline Pseudoartrosis a 6 meses $(n, \%)$ & $2(7,1)$ & $3(I I, I)$ & 0,64 \\
\hline Consolidación $(n, \%)$ & $26(92,9)$ & $24(88,9)$ & 1,04 \\
\hline Falla material osteosíntesis $(n, \%)$ & $0(0)$ & $2(7,4)$ & INDETERMINADO \\
\hline Infección (n,\%) & $2(7,1)$ & $2(7,4)$ & 0,96 \\
\hline Reintervención (n,\%) & $5(17,9)$ & $7(25,9)$ & 0,69 \\
\hline \multicolumn{4}{|l|}{ Tipo de reintervención $(n, \%)$} \\
\hline Lavado $\mathrm{x}$ infección & $2(7, I) 2$ & $(7,4)$ & 0,96 \\
\hline Tratamiento pseudoartrosis & $2(7,1)$ & $3(11, I)$ & 0,64 \\
\hline Retiro material osteosíntesis & I $(3,6)$ & $3(I I, I)$ & 0,32 \\
\hline Corrección mala unión & I $(3,6)$ & $\mathrm{I}(3,7)$ & 0,96 \\
\hline Revisión osteosíntesis & $0(0)$ & $3(11,1)$ & INDETERMINADO \\
\hline Otros & $0(0)$ & I $(3,7)$ & INDETERMINADO \\
\hline
\end{tabular}

* RR - Datos crudos 
a uno corrección por mala unión y a dos tratamiento para el manejo de pseudoartrosis. De los reintervenidos, con PP a dos se les practicó lavado por infección, a tres se les retiró material para revisión quirúrgica, uno para corrección de mala unión y dos por falla de material de osteosíntesis, mientras que a tres se les realizó manejo quirúrgico por pseudoartrosis.

En la evaluación a los seis meses y al primer año postoperatorio el $92,9 \%$ de los pacientes manejados con clavo habían consolidado, mientras que aquellos con placa, el 88,9\% presentaron criterios de consolidación.

\section{Discusión}

Las fracturas metafisiarias distales de tibia muestran un patrón de manejo bastante complicado y complejo. Aunque en los estudios publicados no existe un consenso de tratamiento, se proponen algunos elementos que benefician un procedimiento sobre el otro. La incidencia de consolidación en el estudio fue mayor en los pacientes manejados con CE $(92,9 \%)$ que con PP $(88,9 \%)$ $(\mathrm{RR}=1.04)$. La incidencia de reintervención fue de 17,9\% en los manejados con clavo, contra $25,9 \%$ con placa. Esto evidenciaría una reducción del riesgo del 31\% de requerir reintervención en los manejados con $\mathrm{CE}$ $(\mathrm{RR}=0.69)$. Este porcentaje superior podría estar determinado en esta cohorte por el mayor porcentaje de pseudoartrosis, de falla del material de osteosíntesis y de la discretamente elevada infección en los pacientes que se manejaron con PP. En la literatura se encuentran cifras de reintervención similares, pero por lo regular se asocian más osteosíntesis y CE. ${ }^{2,3}$

El porcentaje de infección fue similar en ambos grupos: $7,1 \%$ con $\mathrm{CE}$, contra $7,4 \%$ de $\mathrm{PP}$, aunque el promedio de tiempo quirúrgico en los pacientes con placa fue 188.75 minutos, bastante mayor al de los dos grupos en general, que fue de 111 minutos. Además llama la atención que este desenlace se presentó en pacientes menores de 50 años, sin diferencia significativa entre fracturas abiertas y cerradas, pero evidenciando un porcentaje importante de pacientes con fracturas Tsherne II (50-66\%) y en menor porcentaje pacientes con fracturas abiertas grados I y II (18-39\%). Esto podría sugerir que la infección en el grupo manejado con PP estaría influenciada más por el tiempo quirúrgico y el estado de los tejidos blandos que por la complejidad de la fractura y la edad de presentación. Estos resultados revelan concordancia con lo reportado en la literatura, en especial con el estudio prospectivo aleatorizado de $\mathrm{Im}^{3}$ donde el índice de infección es superior en el grupo de placa (23\%), en casos de compromiso de tejidos blandos, tiempo quirúrgico mayor que el grupo control (clavo) y un promedio de edad de 40 años.

La incidencia de mala unión fue mayor en los pacientes manejados con CE, tal como se ve en lo reportado por Im, ${ }^{1}$ Zelle, ${ }^{2}$ Vallier ${ }^{9}$ y Nork. ${ }^{10}$ Esto podría verse influenciado por la asociación con fractura ipsilateral del peroné, donde el $60 \%$ de pacientes manejados con CE la presentaron y fueron manejadas con osteosíntesis en el $29 \%$. De estos pacientes que presentaron mala unión, no se les realizó osteosíntesis electiva al peroné. Esto sugeriría una posible indicación errónea dependiente del compromiso de la sindesmosis, como lo reporta Egol, ${ }^{11}$ en pacientes con fracturas inestables de tibia distal asociadas con las de peroné al mismo nivel. Hubo falla del material de osteosíntesis en tres casos manejados con placa $(11,1 \%)$, a los cuales se les realizó revisión de la misma con consolidación al final del seguimiento. Estos datos estarían acordes con lo publicado en los estudios de Collinge ${ }^{12}$ y Borg ${ }^{13}$ donde se alcanzan tasas de falla hasta del 9,5\%.

Este trabajo es el primero realizado en Colombia que compara el manejo de las fracturas diafisiarias distales de tibia, recogiendo la experiencia de los dos centros de referencia citados, con estandarización de las técnicas quirúrgicas en el protocolo del servicio para el seguimiento y con un tamaño de muestra de 55 pacientes, la serie más grande publicada en el país. Es importante anotar que la baja frecuencia de este tipo de fractura impone un período de reclutamiento amplio y la necesidad de controlar las variables en el diseño que reducen la población a estudio. Además, se debe considerar la existencia de algunas variables de confusión que deben controlarse en el análisis aumentado la muestra. Por ahora, la adecuada descripción permite generar hipótesis que apoyadas en la literatura parecerían mostrar mayor frecuencia de mala unión al usar clavo, pero con mejores tasas de consolidación y menos complicaciones y pseudoartrosis. Sin embargo, los resultados deben interpretarse con precau- 
ción pues no se ha realizado análisis ajustado por variables de confusión.

\section{Conclusiones}

Los índices de consolidación son similares a los reportados en la literatura mundial y los de mala unión y pseudoartrosis son un poco menores que en estudios previos. Complicaciones tales como infección y falla de material de osteosíntesis muestran similitud en incidencia con lo publicado. Al hacer una comparación, el manejo con clavo endomedular muestra un índice de mala unión un poco superior que aquellos con placa, pero existe un porcentaje de mayor consolidación y unos de complicaciones y reintervención menores.

\section{Referencias}

1. Schmidt A, Tornetta III P. Treatment of closed tibial fractures. Instr Course Lect. 2003; 52: 607-22.

2. Zelle B, et al. Treatment of distal tibial fractures without articular involvement: A systematic review of 1125 fractures. J Orthop Trauma. 2006; 20: 76-79.

3. Im GI, Tae SK. Distal metaphyseal fractures of tibia: a prospective randomized trial of closed reduction and intramedullary nail versus open reduction and plate and screws fixation. J Trauma 2005; 59:1219-23.

4. Helfet DL, Suk M. Minimally invasive percutaneous plate osteosynthesis of fractures of the distal tibia. Instr Course Lect. 2004; 53:471-5.
5. Borg T, Larsson S. Percutaneous plating of distal tibial fractures. Preliminary results in 21 patients. Injury. 2004; 35: 608-14.

6. Borrelli J. Prickett W. Extraosseous blood supply of the tibia and the effects of different plating techniques: A human cadaveric study. J Orthop Trauma 2002; 16(10): 691-95.

7. Baumgaertel F, Buhl M, Rahn BA. Fracture healing in biological plate osteosynthesis. Injury.1998; 29:3-6.

8. Janssen KW, Biert J, van Kampen A. Treatment of distal tibial fractures: plate versus nail. A retrospective outcome analysis of matched pairs of patients. Int Orthop. 2007 Oct; 31(5):709-14.

9. Vallier HA, Le TT, Bedi A. Radiographic and clinical comparisons of distal tibia shaft fractures $(4$ to $11 \mathrm{~cm}$ proximal to the plafond): plating versus intramedullary nailing. J Orthop Trauma. 2008; 22(5): 307-11.

10. Nork SE, Schwartz A, Agel J. Intramedullary nailing of distal metaphyseal tibial fractures. J Bone Joint Surg Am. 2005; 87(6): 1213-21

11. Egol K, Weisz R. Does fibular plating improve alignment after intramedullary nailing of distal metaphyseal tibia fractures?. J Orthop Trauma 2006; 20(2): 94-103.

12. Collinge C, Kuper M, Larson K, Protzman R. Minimally invasive plating of high-energy metaphyseal distal tibia fractures. J Orthop Trauma. 2007; 21: 355-61.

13. Borg T, Larsson S. Percutaneous plating of distal tibial fractures. Preliminary results in 21 patients. Injury. 2004; 35: 608-14. 